\title{
Non-abelian Vortices: Existence, Uniqueness and Asymptotics
}

\author{
Gabriella Tarantello
}

\begin{abstract}
We provide a unified approach in the (analytical) study of non-abelian vortices for the models proposed in $[4,7]$. We also obtain the necessary estimates to describe the asymptotic behavior of a vortex configuration under a regime of physical interest.
\end{abstract}

Mathematics Subject Classification (2010). Primary 35J20, 35J50, 35J60; Secondary 58J38.

Keywords. Non-abelian vortices, variational methdos, elliptic systems.

\section{Introduction}

In recent years much attention has been devoted to the understanding of non-abelian vortices, also in connection with the delicate issue of confinement. In this respect several models have been proposed (see [1, 2, 5, 8, 10, 14, 15, 17] and references therein) and analyzed in relation with the well understood abelian-Higgs or $U(1)$ model and the corresponding ANO-vortices [9].

Non-abelian vortices concern models that include both abelian and non abelian gauge fields, which may be considered in the framework of a general $G \times U(1)$ gauge theory (typically $G=S O(2 N)$ or $G=U S p(2 N)$ ) as in $[7,2,3,13,16]$, or embedded in a supersymmetric theory as in $[4,5,8]$.

In all cases, the "essential" structure of the abelian model is preserved, and for a special choice of the physical parameters, the second order field equations decouple into first order (selfdual) equations of the BPS-type in matrix form. The desired (non-abelian) vortices are obtained as the static bi-dimensional solutions of the BPS-equations.

However, in contrast to the abelian situation, the non-abelian BPS-vortex equations are difficult to solve analytically, and beside some numerical result, they have been handled only under some a priori ansatz on the structure of the solution (see e.g. [3], [4], [7]).

This work was completed with the support of the FIRB-Ideas project: Analysis and Beyond. 
Here, we shall pursue further the analytical study of non-abelian vortices and provide a unified approach that includes in particular the models introduced in $[4,5,6,8]$ and in $[7,2,3,13,16]$.

In this way we obtain a simple and direct proof of the existence and uniqueness results in $[11,12]$, in addition to some sharp a priori estimates. Those estimates will allow us to determine the asymptotic behavior of the non-abelian vortex solution for limiting values of certain relevant parameters, as useful in the physical applications.

In particular for the models in $[4,5,6,8]$, involving $S U(N)$-gauge fields (see below), we shall be able to describe the asymptotic regime: $N \rightarrow \infty$.

\section{Preliminaries}

In this section we derive the elliptic system of equations that governs non-abelian BPS-vortices (satisfying suitable ansatz) relative to $[4,7]$.

We start with the model in [4] (see also [5, 6]), which is formulated in terms of two gauge fields $(W)_{\mu=0,1,2,3}$ and $(w)_{\mu=0,1,2,3}$ over the groups $U(N)$ and $U(1)$ respectively.

The corresponding $N$-Higgs fields can be identified, with a $N \times N$ complex matrix $H$ (in the fundamental representation). The parameters involved are the $S U(N)$ coupling parameter $g$, the $U(1)$ coupling parameter $e$ and the Fayet-Iliopoulos constant $c$, in terms of which the mass spectra $m_{g}$ and $m_{e}$ for the non-abelian and abelian fields are given respectively as follows:

$$
m_{g}=g \sqrt{c} \text { and } m_{e}=e \sqrt{2 N c} .
$$

Using standard notation, where $I_{N}$ denotes the $N \times N$ identity matrix and $<H>$ denotes the traceless part of the matrix $H$, the BPS-equations relative to static vortex (string) configurations (along the $x_{3}$-axis) take the form:

$$
\frac{1}{2}\left(D_{1} H+i D_{2} H\right)=0, F_{12}=\frac{m_{g}^{2}}{2 c}<H H^{\dagger}>; f_{12}=\frac{m_{g}^{2}}{2 c} \operatorname{Tr}\left(H H^{\dagger}-c I_{N}\right)
$$

where: $D_{j} H=\partial_{j} H+i\left(W_{j}+w_{j} I_{N}\right) H, j=1,2$; the non-abelian flux $F_{12}=\partial_{1} W_{2}-$ $\partial_{2} W_{1}+i\left[W_{1}, W_{2}\right]$ and the abelian flux $f_{12}=\partial_{1} w_{2}-\partial_{2} w_{1}$. All the fields above are supposed to be nontrivial only with respect to the index $\mu=1,2$; see [4,5] for details.

In spite of their "formal" analogy with the abelian case (cfr. [9]), the non-abelian equations (2.2) are much harder to handle analytically, and so far a (radial) solution is obtained only under the ansatz:

$$
H=\sqrt{c} \operatorname{diag}\left(h_{1}, h_{2}, \ldots, h_{2}\right)
$$

with $h_{1}=h_{1}\left(x_{1}, x_{2}\right) \in \mathbb{C}$ and $0<h_{2}=h_{2}\left(x_{1}, x_{2}\right) \in \mathbb{R}$ two smooth functions satisfying (away from the zeroes of $h_{1}$ ):

$$
\left\{\begin{array}{l}
-\Delta \log \left|h_{1}\right|^{2}=m_{e}^{2}-\left(\frac{m_{e}^{2}}{N}+m_{g}^{2} \frac{N-1}{N}\right)\left|h_{1}\right|^{2}-\frac{N-1}{N}\left(m_{e}^{2}-m_{g}^{2}\right) h_{2}^{2} \\
-\Delta \log h_{2}^{2}=m_{e}^{2}-\left(m_{e}^{2} \frac{N-1}{N}+\frac{m_{g}^{2}}{N}\right) h_{2}^{2}-\frac{1}{N}\left(m_{e}^{2}-m_{g}^{2}\right)\left|h_{1}\right|^{2} .
\end{array}\right.
$$


The complex function $h_{1}$ is allowed to vanish at an assigned set of (vortex) points $\left\{p_{1}, \ldots, p_{n}\right\}$ (repeated with multiplicity), so that

$$
\log \left|h_{1}\right|^{2}-\sum_{j=1}^{n} \log \left|x-p_{j}\right|^{2} \in C^{\infty} .
$$

In this way one can check that, by taking $(W)_{\mu=1,2}$ and $(w)_{\mu=1,2}$ satisfying

$$
\frac{1}{2}\left[\left(W_{1}+w_{1} I_{N}\right)+i\left(W_{2}+w_{2} I_{N}\right)\right]=-i S^{-1} \bar{\partial} S
$$

with

$$
c S S^{\dagger}=\operatorname{diag}\left(\left(\frac{\left|h_{1}\right|^{2}}{\Pi_{j}\left|x-p_{j}\right|^{2}}\right)^{-1}, h_{2}^{-2}, \ldots, h_{2}^{-2}\right),
$$

then (2.2) holds, (see [4]).

Furthermore we can recover the fluxes as follows:

$$
\left\{\begin{array}{l}
f_{1,2}=\frac{1}{2 N} \Delta\left(\log \left|h_{1}\right|^{2}+(N-1) \log h_{2}^{2}\right) \\
F_{1,2}=\frac{N-1}{2 N} \Delta\left(\log \left|h_{1}\right|^{2}-\log h_{2}^{2}\right) \operatorname{diag}\left(1,-\frac{1}{N-1}, \ldots,-\frac{1}{N-1}\right) .
\end{array}\right.
$$

Obviously, when $N=1$ the solution behaves exactly as an abelian vortex with the Higgs particle described by a complex function $h$ satisfying (away from the vortex points):

$$
-\Delta \log |h|=m_{e}^{2}\left(1-|h|^{2}\right) .
$$

A similar approach allows one to reduce the search of non-abelian vortices (in the $x_{3}$-direction) for the model proposed in [7] to the study of an analogous elliptic system.

Indeed in [7] the authors show that, when the number of matter flavors equals $2 N$, then it is still possible to describe (non-abelian) vortex configurations as solutions of a (self-dual) BPS equations analogous to (2.2), expressed in terms of the mixed color-flavor $2 N \times 2 N$ matrix $q$ (representing the scalar quarks fields) and the abelian and non-abelian fluxes, see [7].

Actually in [7], to obtain a solution for such BPS-equations, the authors require that the quark fields only wind in the abelian subgroup $(U(1))^{N}$. In this way the matrix $q$ takes the following diagonal form:

$$
q=\operatorname{diag}\left(\phi I_{N}, \psi I_{N}\right)
$$

with $\phi=\phi\left(x_{1}, x_{2}\right)$ and $\psi=\psi\left(x_{1}, x_{2}\right)$ complex functions; and the gauge field $(A)_{\mu=1,2}$ takes the form:

$$
A_{j}=a_{j} I_{N}+b_{j} T ; T=\operatorname{diag}\left(I_{N},-I_{N}\right)
$$

with $a_{j}$ and $b_{j}$ real smooth functions, $j=1,2$. Thus, setting $a=a_{1}+i a_{2}$ and $b=b_{1}+i b_{2}$, the relative BPS-equations are satisfied provided that

$$
\bar{\partial} \phi=\frac{i}{2}(a+b) \phi ; \bar{\partial} \psi=\frac{i}{2}(a-b) \psi
$$


and

$$
\left\{\begin{array}{l}
\partial_{1} a_{2}-\partial_{2} a_{1}=-\frac{e^{2}}{4}\left(|\phi|^{2}+|\psi|^{2}-\frac{v_{0}^{2}}{N}\right) \\
\partial_{1} b_{2}-\partial_{2} b_{1}=-\frac{g^{2}}{4}\left(|\phi|^{2}-|\psi|^{2}\right)
\end{array}\right.
$$

where again $e$ and $g$ denote respectively the abelian and non-abelian coupling parameter, and $v_{0}^{2}$ relates to the symmetry breaking parameter.

On the other hand, we can use (2.8) to express the fluxes $a_{12}=\partial_{1} a_{2}-\partial_{2} a_{1}$ and $b_{12}=\partial_{1} b_{2}-\partial_{2} b_{1}$ as follows:

$$
a_{12}=\frac{1}{4} \Delta\left(\log |\phi|^{2}+\log |\psi|^{2}\right) ; b_{12}=\frac{1}{4} \Delta\left(\log |\phi|^{2}-\log |\psi|^{2}\right) .
$$

So away from the zeroes of $\phi$ and $\psi$, we can formulate $(2.8)-(2.10)$ in the following form:

$$
\left\{\begin{array}{l}
-\Delta \log |\phi|^{2}=\frac{e^{2}+g^{2}}{2}|\phi|^{2}+\frac{e^{2}-g^{2}}{2}|\psi|^{2}-\frac{v_{0}^{2}}{2 N} e^{2} \\
-\Delta \log |\psi|^{2}=\frac{e^{2}+g^{2}}{2}|\psi|^{2}+\frac{e^{2}-g^{2}}{2}|\phi|^{2}-\frac{v_{0}^{2}}{2 N} e^{2} .
\end{array}\right.
$$

The equation (2.8) implies that $\phi$ and $\psi$ may vanish only at single points (i.e. the vortex points) with integral multiplicity.

Following [7], we assume (for simplicity) that $\psi$ never vanishes, while $\phi$ vanishes exactly at the (given) points $\left\{p_{1}, \ldots, p_{n}\right\}$ (repeated according to multiplicity). In other words:

$$
\log |\psi|^{2} \in C^{\infty}\left(\mathbb{R}^{2}\right) ;\left(\log |\phi|^{2}-\sum_{j=1}^{n} \log \left|x-p_{j}\right|^{2}\right) \in C^{\infty}\left(\mathbb{R}^{2}\right) .
$$

As a matter of fact, by taking into account (2.5) and (2.12), we can formulate both (2.4) and (2.11) into the following elliptic system of equations:

$$
\left\{\begin{array}{l}
\Delta v_{1}=(\tau \alpha+(1-\tau) \beta) e^{v_{1}}+(1-\tau)(\alpha-\beta) e^{v_{2}}-\alpha+\sum_{j=1}^{n} \delta_{p_{j}} \\
\Delta v_{2}=(\tau \beta+(1-\tau) \alpha) e^{v_{2}}+\tau(\alpha-\beta) e^{v_{1}}-\alpha
\end{array}\right.
$$

with $\alpha, \beta>0$ and $\tau \in(0,1)$.

Indeed (2.13) reduces to (2.4) simply by setting:

$$
v_{1}=\log \left|h_{1}\right|^{2}, v_{2}=\log h_{2}^{2}, \alpha=m_{e}^{2}, \beta=m_{g}^{2}, \tau=\frac{1}{N}
$$

while $(2.13)$ reduces to $(2.11)$ when

$$
v_{i}=\log \left|\phi_{i}\right|^{2}-\log \frac{v_{0}^{2}}{2 N}, i=1,2, \alpha=\frac{e^{2} v_{0}^{2}}{2 N}, \beta=\frac{g^{2} v_{0}^{2}}{2 N}, \tau=\frac{1}{2} .
$$

Other models for non-abelian vortices can be brought into the elliptic system (2.13). Notice also that for $\alpha=\beta$, the first equation in (2.13) reduces to the familiar ANO-vortex equation (cfr. [9]):

$$
\Delta v=\beta\left(e^{v}-1\right)+4 \pi \sum_{j=1}^{n} \delta_{p_{j}} .
$$


In the next two sections we shall describe the solvability of (2.13) under periodic and topological boundary conditions. In particular, we shall recover the results in $[11,12]$.

\section{Periodic solutions of (2.13)}

Let $\Omega \subset \mathbb{R}^{2}$ be a given periodic cell domain. In this section we discuss the solvability of (2.13) subject to periodic boundary conditions on $\partial \Omega$. More precisely, denote by $G(x, p)$ the Green's function of $\Delta$ under periodic boundary conditions and such that: $\int_{\Omega} G(x, p)=0$. Let

$$
u_{0}(x)=4 \pi \sum_{j=1}^{n} G\left(x, p_{j}\right) .
$$

In the new variables $\left(u_{1}, u_{2}\right): v_{1}=u_{0}+u_{1}$ and $v_{2}=u_{2}$, periodic solution of (2.13) are obtained by solving:

$$
\left\{\begin{array}{l}
\Delta u_{1}=(\tau \alpha+(1-\tau) \beta) e^{u_{0}+u_{1}}+(1-\tau)(\alpha-\beta) e^{u_{2}}-\alpha+\frac{4 \pi N}{|\Omega|} \text { in } \Omega \\
\Delta u_{2}=(\tau \beta+(1-\tau) \alpha) e^{u_{2}}+\tau(\alpha-\beta) e^{u_{0}+u_{1}}-\alpha \text { in } \Omega \\
u_{1} \text { and } u_{2} \text { doubly periodic over } \partial \Omega .
\end{array}\right.
$$

Integrating the above equations over $\Omega$ and by performing simple algebra, we derive that

$$
\int_{\Omega} e^{u_{0}+u_{1}}=|\Omega|-\left(\frac{1-\tau}{\beta}+\frac{\tau}{\alpha}\right) 4 \pi n:=\rho_{1} ; \int_{\Omega} e^{u_{2}}=|\Omega|-\tau\left(\frac{1}{\alpha}-\frac{1}{\beta}\right) 4 \pi n:=\rho_{2}
$$

from which we deduce the following necessary condition for the solvability of (3.1):

$$
\frac{1-\tau}{\beta}+\frac{\tau}{\alpha}<\frac{4 \pi n}{|\Omega|}
$$

On the other hand, by assuming (3.3) and by setting: $u_{j}=w_{j}+c_{j}$ with $\int_{\Omega} w_{j}=$ 0 and $c_{j}=f_{\Omega} w_{j} ; j=1,2$, we find that,

$$
e^{c_{1}}=\frac{\rho_{1}}{\int_{\Omega} e^{u_{0}+w_{1}}} ; e^{c_{2}}=\frac{\rho_{2}}{\int_{\Omega} e^{w_{2}}} \text { with } \rho_{j}>0 \text { in }(3.2)
$$

$j=1,2$ and we can formulate (3.1) only in terms of the unknowns $\left(w_{1}, w_{2}\right)$ as follows:

$$
\left\{\begin{array}{l}
\Delta w_{1}=(\tau \alpha+(1-\tau) \beta) \rho_{1}\left(\frac{e^{u_{0}+w_{1}}}{\int_{\Omega} e^{u_{0}+w_{1}}}-\frac{1}{|\Omega|}\right)+(1-\tau)(\alpha-\beta) \rho_{2}\left(\frac{e^{w_{2}}}{\int_{\Omega} e^{w_{2}}}-\frac{1}{|\Omega|}\right) \text { in } \Omega \\
\Delta w_{2}=(\tau \beta+(1-\tau) \alpha) \rho_{2}\left(\frac{e^{w_{2}}}{\int_{\Omega} e^{w_{2}}}-\frac{1}{|\Omega|}\right)+\tau(\alpha-\beta) \rho_{1}\left(\frac{e^{u_{0}+w_{1}}}{\int_{\Omega} e^{u_{0}+w_{1}}}-\frac{1}{|\Omega|}\right) \text { in } \Omega \\
\int_{\Omega} w_{1}=0, \int_{\Omega} w_{2}=0, w_{1} \text { and } w_{2} \text { doubly periodic over } \partial \Omega .
\end{array}\right.
$$

We are going to obtain an equivalent formulation of (3.5) that has the advantage to admit a variational formulation in the space $E=\mathcal{H}(\Omega) \times \mathcal{H}(\Omega)$, where the Hilbert space: $\mathcal{H}(\Omega)=\left\{w \in H_{\text {loc }}^{1}\left(\mathbb{R}^{2}\right): w\right.$ is periodic with cell domain $\Omega$ and $\left.\int_{\Omega} w=0\right\}$ is equipped with the usual scalar product. 
Observe that by straightforward calculations we have:

$$
\left\{\begin{array}{l}
\Delta\left(\tau w_{1}+(1-\tau) w_{2}\right)=\alpha\left[\tau \rho_{1}\left(\frac{e^{u_{0}+w_{1}}}{\int_{\Omega} e^{u_{0}+w_{1}}}-\frac{1}{|\Omega|}\right)+(1-\tau) \rho_{2}\left(\frac{e^{w_{2}}}{\int_{\Omega} e^{w_{2}}}-\frac{1}{|\Omega|}\right)\right] \text { in } \Omega \\
\Delta\left(w_{1}-w_{2}\right)=\beta\left[\rho_{1}\left(\frac{e^{u_{0}+w_{1}}}{\int_{\Omega} e^{u_{0}+w_{1}}}-\frac{1}{|\Omega|}\right)-\rho_{2}\left(\frac{e^{w_{2}}}{\int_{\Omega} e^{w_{2}}}-\frac{1}{|\Omega|}\right)\right] \text { in } \Omega \\
\left(w_{1}, w_{2}\right) \in E .
\end{array}\right.
$$

Thus, in terms of the new unknowns:

$$
z_{1}=\tau w_{1}+(1-\tau) w_{2} \text { and } z_{2}=w_{1}-w_{2}
$$

we easily check that (weak) solutions of (3.6) correspond to critical points of the following functional:

$$
\begin{aligned}
I\left(z_{1}, z_{2}\right)= & \frac{1}{2 \alpha}\left\|\nabla z_{1}\right\|_{2}^{2}+\frac{\tau(1-\tau)}{2 \beta}\left\|\nabla z_{2}\right\|_{2}^{2} \\
& +\tau \rho_{1} \log f_{\Omega}\left(e^{u_{0}+z_{1}+(1-\tau) z_{2}}\right)+(1-\tau) \rho_{2} \log f_{\Omega}\left(e^{z_{1}-\tau z_{2}}\right)
\end{aligned}
$$

with $\left(z_{1}, z_{2}\right) \in E$.

Proposition 3.1. If $\tau \in(0,1), \alpha>0, \beta>0$ and $\rho_{j}>0, j=1,2$, then the functional $I$ admits a unique critical point corresponding to its global minimizer.

Proof. By Jensen inequality we have:

$$
f_{\Omega}\left(e^{u_{0}+z_{1}+(1-\tau) z_{2}}\right) \geq 1 \text { and } f_{\Omega}\left(e^{z_{1}-\tau z_{2}}\right) \geq 1
$$

Thus, we find that $I$ is coercive, bounded from below, lower semicontinuous and strictly convex. Therefore it attains its minimum value at (exactly) one point that defines its only possible critical point.

Notice in particular that if $\left(z_{1}, z_{2}\right): I\left(z_{1}, z_{2}\right)=\min _{E} I$, then $I\left(z_{1}, z_{2}\right) \leq I(0,0)$. As a consequence we obtain:

$$
\begin{gathered}
\tau(1-\tau)\left\|\nabla z_{2}\right\|_{2}^{2}+\left\|\nabla z_{1}\right\|_{2}^{2} \leq 2 \tau \max \{\alpha, \beta\} \rho_{1} \log \left(f_{\Omega} e^{u_{0}}\right) \\
0 \leq \log \int_{\Omega} e^{z_{1}-\tau z_{2}} \leq \frac{\tau \rho_{1}}{(1-\tau) \rho_{2}} \log \left(f_{\Omega} e^{u_{0}}\right) \\
\int_{\Omega} e^{u_{0}+z_{1}+(1-\tau) z_{2}} \leq \int_{\Omega} e^{u_{0}}
\end{gathered}
$$

In particular, in terms of the periodic problem (3.1) we may conclude the following:

Theorem 3.2. The condition (3.3) is necessary and sufficient for the solvability of (3.1), and if (3.3) holds, then (3.1) admits a unique solution $\left(u_{1}, u_{2}\right)$. Moreover letting: $c_{j}=f_{\Omega} u_{j}$ and $w_{j}=u_{j}-c_{j}, j=1,2$, then $c_{j}=c_{j}\left(w_{j}\right)$ satisfies (3.4), and there exists a constant $C>0$, independent of $\tau, \alpha, \beta$ such that:

$$
\text { i) } \tau\left\|\nabla w_{1}\right\|_{2}^{2}+(1-\tau)\left\|\nabla w_{2}\right\|_{2}^{2} \leq C \tau \max \{\alpha, \beta\} \rho_{1} ;\left\|\nabla\left(w_{1}-w_{2}\right)\right\|_{2}^{2} \leq \frac{C \beta \rho_{1}}{1-\tau}
$$




$$
\text { ii) } 1 \leq f_{\Omega} e^{u_{0}+w_{1}} \leq C ; \log \left(f_{\Omega} e^{w_{2}}\right) \leq C \frac{\tau \rho_{1}}{(1-\tau) \rho_{2}}
$$

with $\rho_{1}>0, \rho_{2}>0$ defined by $(3.2)$.

Clearly the estimates in Theorem 3.2 allow us to handle easily the asymptotic regime:

$$
\gamma=\frac{\beta}{\alpha} \rightarrow 1, \tau \in(0,1) \text { fixed }
$$

where, without surprise, we find that: $w_{1} \rightarrow w_{A}, w_{2} \rightarrow 0$ in $\mathcal{H}(\Omega)$ (in any relevant norm), and $w_{A}$ is the unique solution of the abelian periodic vortex equation:

$$
\left\{\begin{array}{l}
\Delta w=\left(\beta-\frac{4 \pi n}{|\Omega|}\right)\left(\frac{e^{u_{0}+w}}{\int_{\Omega} e^{u_{0}+w}}-1\right) \text { in } \Omega \\
w \in \mathcal{H}(\Omega)
\end{array}\right.
$$

with $\beta \in\left(\frac{4 \pi n}{|\Omega|},+\infty\right)$ (this expresses the necessary condition $(3.3)$ when $\alpha=\beta$ ).

Notice in particular that the parameter $\tau \in(0,1)$ plays no role in the asymptotic regime $(3.12)$.

More interestingly, we can show an analogous behavior for the less obvious asymptotic regime:

$$
\beta \in\left(\frac{4 \pi n}{|\Omega|},+\infty\right) \text { and } \alpha \tau=m_{0}>0 \text { fixed, but } \alpha \rightarrow+\infty, \tau \rightarrow 0
$$

that in the context of (2.4) (see (2.1) and (2.14)) corresponds to the asymptotic situation of physical interest: $N \rightarrow \infty$ and $\gamma \rightarrow 0$.

In view of the estimates i) and ii) of Theorem 3.2, in the asymptotic regime (3.14), we still find:

$$
w_{1} \rightarrow w_{A} \text { and } w_{2} \rightarrow 0 .
$$

Indeed (3.15) certainly holds along a sequence, and the convergence is weak in $\mathcal{H}(\Omega)$, pointwise a.e. in $\Omega$ and strongly in $L^{p}(\Omega), \forall p \geq 1$, with $w_{A}$ satisfying (3.13), as it follows by using the second equation in (3.6).

The uniqueness of the limit ensures that the convergence actually holds for the full solution set (not only along sequences) and in strong norms. To check the strong convergence, we use the second equation in (3.5), that we multiply by $\left(w_{2}-\log f_{\Omega} e^{w_{2}}\right)$ and integrate over $\Omega$ to obtain:

$$
\begin{aligned}
\left\|\nabla w_{2}\right\|_{2}^{2}= & ((1-\tau) \alpha+\tau \beta) \frac{\rho_{2}}{|\Omega|}\left(1-e^{w_{2}-\log f_{\Omega} e^{w_{2}}}\right)\left(w_{2}-\log f_{\Omega} e^{w_{2}}\right) \\
& +\tau(\alpha-\beta) \frac{\rho_{1}}{|\Omega|}\left(1-\frac{e^{u_{0}+w_{1}}}{f_{\Omega} e^{u_{0}+w_{1}}}\right)\left(w_{2}-\log f_{\Omega} e^{w_{2}}\right) \\
\leq & \tau \alpha\left|1-\frac{\beta}{\alpha}\right| \frac{\rho_{1}}{|\Omega|}\left(\left\|w_{2}-\log f_{\Omega} e^{w_{2}}\right\|_{1}+\frac{\left\|e^{u_{0}+w_{1}}\right\|_{2}}{f_{\Omega} e^{u_{0}+w_{1}}}\left\|w_{2}-\log f_{\Omega} e^{w_{2}}\right\|_{2}\right) .
\end{aligned}
$$

Thus, in the asymptotic regime (3.14), the estimates i) and ii) together with the Moser-Trudinger inequality (cfr. e.g. [18]), allow us to obtain a constant $C>0$ : $\left\|\nabla w_{2}\right\|_{2}^{2} \leq C\left\|w_{2}-\log f_{\Omega} e^{w_{2}}\right\|_{2} \rightarrow 0$. We can use this information in the second equation of (3.6) to get also the strong convergence of $w_{1}$ towards $w_{A}$. 


\section{Topological solutions of (2.13)}

In this section we are going to study $(2.13)$ in $\mathbb{R}^{2}$, under the topological boundary condition:

$$
v_{j}(x) \rightarrow 0 \text { as }|x| \rightarrow \infty, \quad j=1,2 .
$$

To this purpose, for given $\mu>0$, we introduce the function:

$$
u_{0}(x)=\sum_{j=1}^{n} \log \left(\frac{\left|x-p_{j}\right|^{2}}{\mu+\left|x-p_{j}\right|^{2}}\right)
$$

so that in the new (smooth) variables:

$$
u_{1}=v_{1}-u_{0}, u_{2}=v_{1}
$$

we are reduced to analyze the following problem:

$$
\left\{\begin{array}{l}
-\Delta u_{1}=(\tau \alpha+(1-\tau) \beta)\left(1-e^{u_{o}+u_{1}}\right)+(1-\tau)(\alpha-\beta)\left(1-e^{u_{2}}\right)-g_{0} \\
-\Delta u_{2}=(\tau \beta+(1-\tau) \alpha)\left(1-e^{u_{2}}\right)+\tau(\alpha-\beta)\left(1-e^{u_{o}+u_{1}}\right) \\
u_{j}(x) \rightarrow 0 \text { as }|x| \rightarrow \infty
\end{array}\right.
$$

with $g_{0}(x)=4 \sum_{j=1}^{n} \frac{\mu}{\left(\mu+\left|x-p_{j}\right|^{2}\right)^{2}} \in L^{1}\left(\mathbb{R}^{2}\right) \cap L^{\infty}\left(\mathbb{R}^{2}\right)$.

As above, when $\alpha=\beta$ the solution $\left(u_{1}, u_{2}\right)$ reduces simply to: $u_{1}=u_{A}, u_{2}=0$; where $u_{A}$ is the (unique) topological solution of the abelian vortex problem:

$$
\left\{\begin{array}{l}
-\Delta u=\beta\left(1-e^{u_{o}+u}\right)-g_{0} \\
u(x) \rightarrow 0 \text { as }|x| \rightarrow \infty
\end{array}\right.
$$

Actually, most of the properties valid for (4.3) are satisfied by solution of (4.2).

Lemma 4.1. Let $\left(u_{1}, u_{2}\right)$ be a solution for (4.2). For $\alpha \neq \beta$ we have:

$$
\begin{gathered}
\text { a) } u_{0}+u_{1}<0 \text { and }(\alpha-\beta) u_{2}>0 \text { in } \mathbb{R}^{2} \\
\text { b) }\left(u_{1}, u_{2}\right) \in H^{1}\left(\mathbb{R}^{2}\right) \times H^{1}\left(\mathbb{R}^{2}\right) ;\left(1-e^{u_{0}+u_{1}}\right),\left(1-e^{u_{2}}\right) \in L^{1}\left(\mathbb{R}^{2}\right)
\end{gathered}
$$

and the following holds:

$$
\int_{\mathbb{R}^{2}}\left(1-e^{u_{0}+u_{1}}\right)=\frac{4 \pi n}{\alpha \beta}((1-\tau) \alpha+\tau \beta):=\lambda_{1}, \int_{\mathbb{R}^{2}}\left|1-e^{u_{2}}\right|=\frac{4 \pi n \tau}{\alpha \beta}|\alpha-\beta|:=\lambda_{2}
$$

c) $\left(u_{1}, u_{2}\right)$ decay exponentially fast to zero at infinity.

Proof. To establish a) we only need to show that $u_{0}+u_{1} \leq 0$ in $\mathbb{R}^{2}$, since the strong maximum principle and the second equation in (4.2) will readily yield to the full statement in (4.4). We start with the case $\alpha>\beta$, and argue by contradiction. So we assume there exists $x_{0} \in \mathbb{R}^{2}$ such that: $v_{1}\left(x_{0}\right)=u_{0}\left(x_{0}\right)+u_{1}\left(x_{0}\right)>0$, hence necessarily $x_{0} \notin\left\{p_{1}, \ldots, p_{n}\right\}$.

This fact and (4.1) ensure that there exists $x_{1} \in \mathbb{R}^{2} \backslash\left\{p_{1}, \ldots, p_{n}\right\}: v_{1}\left(x_{1}\right)=$ $\max _{\mathbb{R}^{2}} v_{1}>0$. Then from the first equation in $(2.13)$ we find $v_{2}\left(x_{1}\right)<0$. This allows to obtain $x_{2} \in \mathbb{R}^{2}: v_{2}\left(x_{2}\right)=\min _{\mathbb{R}^{2}} v_{2}<0$ and from the second equation in (2.13), deduce $v_{1}\left(x_{2}\right)>0$.

As a consequence we also know that $v_{1}-v_{2}$ takes positive values and we can define $\bar{x} \in \mathbb{R}^{2} \backslash\left\{p_{1}, \ldots, p_{n}\right\}: v_{1}(\bar{x})-v_{2}(\bar{x})=\max _{\mathbb{R}^{2}} v_{1}-v_{2}$. 
Since $v_{1}(\bar{x})-v_{2}(\bar{x}) \geq v_{1}\left(x_{1}\right)-v_{2}\left(x_{1}\right)=\max _{\mathbb{R}^{2}} v_{1}-v_{2}\left(x_{1}\right)$, we get $v_{2}(\bar{x}) \leq$ $v_{2}\left(x_{1}\right)<0$. Similarly, $v_{1}(\bar{x})-v_{2}(\bar{x}) \geq v_{1}\left(x_{2}\right)-v_{2}\left(x_{2}\right)=v_{1}\left(x_{2}\right)-\min _{\mathbb{R}^{2}} v_{2}$ and so $v_{1}(\bar{x}) \geq v_{1}\left(x_{2}\right)>0$.

But this yield to the following contradiction:

$$
0 \leq-\Delta\left(v_{1}-v_{2}\right)(\bar{x})=\beta\left(1-e^{v_{1}(\bar{x})}\right)-\beta\left(1-e^{v_{2}(\bar{x})}\right)<0 .
$$

In case $\beta>\alpha$ we proceed in analogous way only that now our contradiction assumption yields to the existence of $x_{1}, x_{2} \in \mathbb{R}^{2}: v_{1}\left(x_{1}\right)=\max _{\mathbb{R}^{2}} v_{1}>0$ and $v_{2}\left(x_{1}\right)>0 ; v_{2}\left(x_{2}\right)=\max _{\mathbb{R}^{2}} v_{2}>0$ and $v_{1}\left(x_{2}\right)>0$.

Therefore we find $\hat{x} \in \mathbb{R}^{2} \backslash\left\{p_{1}, \ldots, p_{n}\right\}: \tau v_{1}(\hat{x})+(1-\tau) v_{2}(\hat{x})=\max _{\mathbb{R}^{2}} \tau v_{1}+$ $(1-\tau) v_{2}$ and as above we have: $v_{1}(\hat{x}) \geq v_{1}\left(x_{2}\right)>0$ and $v_{2}(\hat{x}) \geq v_{2}\left(x_{1}\right)>0$.

But this is impossible, since

$$
0 \leq-\Delta\left(\tau v_{1}+(1-\tau) v_{2}\right)(\bar{x})=\alpha\left(\tau\left(1-e^{v_{1}(\bar{x})}\right)+(1-\tau)\left(1-e^{v_{2}(\bar{x})}\right)\right)<0 .
$$

Part b) and c) follow as for the abelian case $\alpha=\beta$, only with obvious modifications, see the proof of Proposition 5 and 6 of [19] and Proposition 3.2.4 of [18].

In analogy with the periodic case, it is easy to show that also (4.2) admits a variational formulation in the space $H^{1}\left(\mathbb{R}^{2}\right) \times H^{1}\left(\mathbb{R}^{2}\right)$ according to the new variables

$$
z_{1}=\tau u_{1}+(1-\tau) u_{2} \text { and } z_{2}=u_{1}-u_{2} .
$$

Indeed, by a straightforward calculation we see that solutions of (4.2) correspond (via (4.6)) to critical points of the functional:

$$
\begin{aligned}
& J\left(z_{1}, z_{2}\right)=\frac{1}{2 \alpha}\left\|\nabla z_{1}\right\|_{2}^{2}+\frac{\tau(1-\tau)}{2 \beta}\left\|\nabla z_{2}\right\|_{2}^{2} \\
& +\tau \int_{\mathbb{R}^{2}}\left(e^{u_{0}}-1\right)\left(e^{z_{1}+(1-\tau) z_{2}}-1\right) d x+\tau \int_{\mathbb{R}^{2}}\left(e^{z_{1}+(1-\tau) z_{2}}-1-\left(z_{1}+(1-\tau) z_{2}\right)\right) d x \\
& +(1-\tau) \int_{\mathbb{R}^{2}}\left(e^{z_{1}-\tau z_{2}}-1-\left(z_{1}-\tau z_{2}\right)\right) d x+\tau \int_{\mathbb{R}^{2}} g_{0}(x)\left(z_{1}+(1-\tau) z_{2}\right) d x .
\end{aligned}
$$

Notice that, $\left(1-e^{u_{0}}\right)$ and $g_{0} \in L^{2}\left(\mathbb{R}^{2}\right)$, while by well known estimates (see e.g. the Appendix in $[9]):\left(e^{z}-1\right) \in L^{2}\left(\mathbb{R}^{2}\right)$ and $\left(e^{z}-1-z\right) \in L^{1}\left(\mathbb{R}^{2}\right)$ for $z \in H^{1}\left(\mathbb{R}^{2}\right)$.

Thus the functional $J \in C^{1}\left(H^{1}\left(\mathbb{R}^{2}\right) \times H^{1}\left(\mathbb{R}^{2}\right)\right)$ is lower semicontinuous and strictly convex in $H^{1}\left(\mathbb{R}^{2}\right) \times H^{1}\left(\mathbb{R}^{2}\right)$.

But now it is less obvious to check that $J$ is also coercive and bounded from below.

To this purpose observe that, if we let for instance $\beta>\alpha$, then we can re-write $J$ as follows:

$$
J\left(z_{1}, z_{2}\right)=\frac{1}{2}\left(\frac{1}{\alpha}-\frac{1}{\beta}\right)\left\|\nabla z_{1}\right\|_{2}^{2}+\tau J_{1}^{\beta}\left(z_{1}+(1-\tau) z_{2}\right)+(1-\tau) J_{2}^{\beta}\left(z_{1}-\tau z_{2}\right),
$$

with

and

$$
J_{1}^{\beta}(v)=\frac{1}{2 \beta}\|\nabla v\|_{2}^{2}+\int_{\mathbb{R}^{2}} e^{u_{0}}\left(e^{v}-1-v\right)+\int_{\mathbb{R}^{2}}\left(e^{u_{0}}-1+g_{0}\right) v
$$

$$
J_{2}^{\beta}(v)=\frac{1}{2 \beta}\|\nabla v\|_{2}^{2}+\int_{\mathbb{R}^{2}}\left(e^{v}-1-v\right), v \in H^{1}\left(\mathbb{R}^{2}\right)
$$


(note that $J_{1}^{\beta}$ reduces to $J_{2}^{\beta}$ when $u_{0} \equiv 0 \equiv g_{0}$ ). Therefore we can apply Proposition 13 in [19] and conclude the existence of positive constants $a, b: J_{1}^{\beta}(v) \geq a\|v\|_{H^{1}}-b$ and $J_{2}^{\beta}(v) \geq a\|v\|_{H^{1}}-b, \forall v \in H^{1}\left(\mathbb{R}^{2}\right)$.

This ensures that, if $\alpha<\beta$, then $J$ is coercive and bounded from below in $H^{1}\left(\mathbb{R}^{2}\right) \times H^{1}\left(\mathbb{R}^{2}\right)$.

On the other hand, when $\alpha>\beta$, then

$J\left(z_{1}, z_{2}\right)=\frac{\tau(1-\tau)}{2}\left(\frac{1}{\alpha}-\frac{1}{\beta}\right)\left\|\nabla z_{2}\right\|_{2}^{2}+\tau J_{1}^{\alpha}\left(z_{1}+(1-\tau) z_{2}\right)+(1-\tau) J_{2}^{\alpha}\left(z_{1}-\tau z_{2}\right)$

where $J_{1}^{\alpha}$ and $J_{2}^{\alpha}$ are the same functionals above only with $\beta$ replaced by $\alpha$. So, also in this case $J$ is coercive and bounded from below.

In any case $J$ admits a unique critical point corresponding to its global minimum. We conclude:

Theorem 4.2. Problem (4.2) admits a unique solution. When expressed in the variables $\left(z_{1}, z_{2}\right)$ in (4.6), it corresponds to the global minimizer of $J$ in $H^{1}\left(\mathbb{R}^{2}\right) \times$ $H^{1}\left(\mathbb{R}^{2}\right)$.

As for the periodic case, we wish to describe the behavior of the solution of (4.2) in the asymptotic regime:

$$
\tau \rightarrow 0, \alpha \rightarrow+\infty \text { but } \tau \alpha=m_{0}>0 \text { and } \beta>0 \text { fixed, }
$$

so that $\gamma=\frac{\beta}{\alpha} \rightarrow 0$. Observe once more that in the context of $(2.4)$, the regime $(4.8)$ corresponds to the interesting physical limit: $N \rightarrow+\infty$.

To this purpose, we shall need the following a priori estimates:

Proposition 4.3. Let $\left(u_{1}, u_{2}\right) \in H^{1}\left(\mathbb{R}^{2}\right) \times H^{1}\left(\mathbb{R}^{2}\right)$ be a solutions of $(4.2)$, and let the values $\lambda_{1}\left(=\lambda_{1}(\alpha, \beta, \tau)\right)$ and $\lambda_{2}\left(=\lambda_{2}(\alpha, \beta, \tau)\right)$ be defined in (4.5). We have:

a) $u_{2} \in L^{1}\left(\mathbb{R}^{2}\right) \cap L^{\infty}\left(\mathbb{R}^{2}\right)$ and there holds:

$$
\begin{gathered}
\left\|u_{2}\right\|_{\infty} \leq \log \left(1+\frac{\tau|\alpha-\beta|}{\alpha-\tau(\alpha-\beta)_{+}}\right) \\
\left\|\nabla u_{2}\right\|_{2}^{2} \leq \lambda_{1} \tau|\alpha-\beta|\left\|u_{2}\right\|_{\infty} .
\end{gathered}
$$

b)

$$
\begin{gathered}
\left\|\nabla u_{1}^{+}\right\|_{2}^{2}+\frac{1}{2}(\tau \alpha+(1-\tau) \beta)\left\|u_{1}^{+}\right\|_{2}^{2} \leq \frac{1}{2}(\tau \alpha+(1-\tau) \beta)\left\|u_{0}\right\|_{2}^{2} \\
\left\|u_{1}^{-}\right\|_{2}^{2} \leq 2 \lambda_{1}\left(1+2\left\|\nabla u_{1}^{-}\right\|_{2}^{2}\right) \\
\left\|\nabla u_{1}^{-}\right\|_{2}^{2} \leq A\left(1+\sqrt{1+\frac{1}{A}}\right)
\end{gathered}
$$

with $A=2 \lambda_{1}\left((1-\tau)|\alpha-\beta|\left(\lambda_{2} e^{\left\|u_{2}\right\|_{\infty}}\right)^{\frac{1}{2}}+\left\|g_{0}\right\|_{2}\right)^{2}$. 
Proof. We start to establish a) in case $\alpha>\beta$. Then $u_{2}>0$ in $\mathbb{R}^{2}$ (by part a) of Lemma 4.1 ) and there exists $x_{2} \in \mathbb{R}^{2}: u_{2}\left(x_{2}\right)=\max _{\mathbb{R}^{2}} u_{2}$. Consequently,

$$
0 \geq \Delta u_{2}\left(x_{2}\right)=((1-\tau) \alpha+\tau \beta)\left(e^{u_{2}\left(x_{2}\right)}-1\right)+\tau(\alpha-\beta)\left(e^{\left(u_{0}+u_{1}\right)\left(x_{2}\right)}-1\right) .
$$

Thus

$$
e^{\left\|u_{2}\right\|_{L} \infty}=e^{u_{2}\left(x_{2}\right)} \leq 1+\frac{\tau(\alpha-\beta)}{(1-\tau) \alpha+\tau \beta}
$$

and (4.9) is established in this case.

In case $\alpha<\beta$, then $u_{2}<0$ and there exists $x_{0} \in \mathbb{R}^{2}: u_{2}\left(x_{0}\right)=\min _{\mathbb{R}^{2}} u_{2}$. In addition,

$$
0 \leq \Delta u_{2}\left(x_{0}\right)=((1-\tau) \alpha+\tau \beta)\left(e^{u_{2}\left(x_{0}\right)}-1\right)-\tau(\alpha-\beta)\left(e^{\left(u_{0}+u_{1}\right)\left(x_{0}\right)}-1\right)
$$

and we derive

$$
e^{-\left\|u_{2}\right\|_{L^{\infty}}}=e^{u_{2}\left(x_{0}\right)} \geq 1-\frac{\tau(\alpha-\beta)}{(1-\tau) \alpha+\tau \beta}=\frac{\alpha}{(1-\tau) \alpha+\tau \beta}
$$

and (4.9) is established in this case as well.

To obtain (4.10) we multiply the second equation in (4.2) by $u_{2}$ and integrate over $\mathbb{R}^{2}$. By the usual integration by parts we derive:

$$
\begin{aligned}
\left\|\nabla u_{2}\right\|_{2}^{2} & =((1-\tau) \alpha+\tau \beta) \int_{\mathbb{R}^{2}}\left(1-e^{u_{2}}\right) u_{2}+\tau(\alpha-\beta) \int_{\mathbb{R}^{2}}\left(1-e^{u_{0}+u_{1}}\right) u_{2} \\
& \leq \tau|\alpha-\beta|\left\|u_{2}\right\|_{\infty} \int_{\mathbb{R}^{2}}\left(1-e^{u_{0}+u_{1}}\right)
\end{aligned}
$$

and in view of (4.5) we deduce (4.10).

Concerning $u_{1}$, we use the first equation in (4.2) to find

$$
-\Delta u_{1}+((1-\tau) \beta+\tau \alpha) u_{1}=((1-\tau) \beta+\tau \alpha)\left(1+u_{1}-e^{u_{0}+u_{1}}\right)-(1-\tau)|\alpha-\beta|\left|1-e^{u_{2}}\right|-g_{0}
$$

that we can multiply by $u_{1}^{+}$and integrate over $\mathbb{R}^{2}$ to obtain:

$$
\left\|\nabla u_{1}^{+}\right\|_{2}^{2}+(\tau \alpha+(1-\tau) \beta)\left\|u_{1}^{+}\right\|_{2}^{2} \leq(\tau \alpha+(1-\tau) \beta)\left\|u_{0}\right\|_{2}\left\|u_{1}^{+}\right\|_{2}
$$

from which (4.11) easily follows.

To establish (4.11) and (4.13), we use the first identity in (4.5) to obtain:

$$
\lambda_{1}=\int_{\mathbb{R}^{2}}\left(1-e^{u_{0}+u_{1}}\right) \geq \int_{\left\{u_{1} \leq 0\right\}}\left(1-e^{u_{0}-u_{1}^{-}}\right) \geq \int_{\mathbb{R}^{2}}\left(1-e^{-u_{1}^{-}}\right),
$$

where in the last inequality we have used that $u_{0}<0$ in $\mathbb{R}^{2}$.

By virtue of the inequality: $\frac{t}{1+t} \leq\left(1-e^{-t}\right) \forall t \geq 0$, we get:

$$
\int_{\mathbb{R}^{2}}\left(\frac{u_{1}^{-}}{1+u_{1}^{-}}\right) \leq \int_{\mathbb{R}^{2}}\left(1-e^{-u_{1}^{-}}\right) \leq \lambda_{1} .
$$

On the other hand, for every $u \in H^{1}\left(\mathbb{R}^{2}\right)$ the following estimate holds:

$$
\|u\|_{2}^{2} \leq 2\left(1+\|\nabla u\|_{2}^{2}\right) \int_{\mathbb{R}^{2}}\left(\frac{|u|}{1+|u|}\right)^{2}
$$

(for the proof see e.g. p. 139-140 of [19]), and (4.12) easily follows from (4.14) and (4.15). 
Concerning (4.13), we use again the second equation in (4.2), which now we multiply by $u_{1}^{-}$, integrate over $\mathbb{R}^{2}$ and get:

$$
\begin{aligned}
\left\|\nabla u_{1}^{-}\right\|_{2}^{2} & =(\tau \alpha+(1-\tau) \beta) \int_{\mathbb{R}^{2}}\left(e^{u_{0}+u_{1}}-1\right) u_{1}^{-}+(1-\tau) \int_{\mathbb{R}^{2}}\left|e^{u_{2}}-1\right| u_{1}^{-}+\int_{\mathbb{R}^{2}} g_{0} u_{1}^{-} \\
& \leq\left((1-\tau)|\alpha-\beta|\left\|e^{u_{2}}-1\right\|_{2}+\left\|g_{0}\right\|_{2}\right)\left\|u_{1}^{-}\right\|_{2} .
\end{aligned}
$$

Since we can estimate:

$$
\left\|e^{u_{2}}-1\right\|_{2} \leq e^{\left\|u_{2}\right\|_{\infty}} \int_{\mathbb{R}^{2}}\left|e^{u_{2}}-1\right|=e^{\left\|u_{2}\right\|_{\infty}} \lambda_{2}
$$

we find:

$$
\left\|\nabla u_{1}^{-}\right\|_{2}^{2} \leq\left((1-\tau)|\alpha-\beta|\left(\lambda_{2} e^{\left\|u_{2}\right\|_{\infty}}\right)^{\frac{1}{2}}+\left\|g_{0}\right\|_{2}\right)\left\|u_{1}^{-}\right\|_{2} .
$$

At this point we can insert in (4.16) the estimate (4.12) that we have already established for $\left\|u_{1}^{-}\right\|_{2}$, and by simple algebra arrive at (4.13).

With the estimate of Proposition 4.3 we can establish the following:

Theorem 4.4. Under the asymptotic regime (4.8) we have:

$$
u_{1} \rightarrow u_{A}, u_{2} \rightarrow 0 \text { in } H^{1}\left(\mathbb{R}^{2}\right)
$$

with $u_{A}$ the unique solution of (4.3).

Proof. By the estimate (4.9) and (4.10) we easily see that, under (4.8), we have

$$
\|u\|_{\infty} \rightarrow 0 \text { and }\left\|\nabla u_{2}\right\|_{2} \rightarrow 0 .
$$

Since, under (4.8) we can assume $\alpha>\beta$, we have: $u_{2}>0$ and

$$
\left\|u_{2}\right\|_{L^{1}} \leq \int_{\mathbb{R}^{2}}\left(e^{u_{2}}-1\right) \rightarrow 0 .
$$

So by using (4.17) together with (4.18), we conclude that $\left\|u_{2}\right\|_{L^{2}} \rightarrow 0$. In other words, $u_{2} \rightarrow 0$ in $H^{1}\left(\mathbb{R}^{2}\right)$ as claimed.

Concerning $u_{1}$, the estimates (4.11), (4.13) and (4.12) give that $u_{1}$ is uniformly bounded in $H^{1}\left(\mathbb{R}^{2}\right)$. Therefore along a sequence we can assume that $u_{1} \rightarrow u_{*} \in$ $H^{1}\left(\mathbb{R}^{2}\right)$, weakly in $H^{1}\left(\mathbb{R}^{2}\right)$, strongly in $L_{\text {loc }}^{2}\left(\mathbb{R}^{2}\right)$ and pointwise a.e. in $\mathbb{R}^{2}$.

On the other hand, recalling the equation for $u_{1}-u_{2}$, namely:

$$
-\Delta\left(u_{1}-u_{2}\right)=\beta\left(1-e^{u_{0}+u_{1}}\right)-\beta\left(1-e^{u_{2}}\right)-g_{0} ;
$$

by taking the limit, we find that necessarily: $u_{*}=u_{A}$ the unique solution to (4.3). Furthermore from (4.19) and (4.3) we also get:

$$
\begin{aligned}
& \left\|\nabla\left(u_{1}-u_{A}\right)\right\|_{2}^{2}+\beta \int_{\mathbb{R}^{2}} e^{u_{0}+u_{A}}\left(e^{u_{1}-u_{A}}-1\right)\left(u_{1}-u_{A}\right) \\
& \leq \int_{\mathbb{R}^{2}} \nabla u_{2} \cdot \nabla\left(u_{1}-u_{A}\right)+\beta \int_{\mathbb{R}^{2}}\left(e^{u_{2}}-1\right)\left(u_{1}-u_{A}\right)-\int_{\mathbb{R}^{2}} g_{0}\left(u_{1}-u_{A}\right) \rightarrow 0 .
\end{aligned}
$$

Hence, $\left\|\nabla\left(u_{1}-u_{A}\right)\right\|_{2}^{2} \rightarrow 0$ and $\int_{\mathbb{R}^{2}} e^{u_{0}+u_{A}}\left(e^{u_{1}-u_{A}}-1\right)\left(u_{1}-u_{A}\right) \rightarrow 0$.

In particular, $\int_{\mathbb{R}^{2}} e^{u_{0}+u_{A}}\left(\left(u_{1}-u_{A}\right)^{+}\right)^{2} \rightarrow 0$ and $\int_{\mathbb{R}^{2}} e^{u_{0}+u_{A}} \frac{\left(\left(u_{1}-u_{A}\right)^{-}\right)^{2}}{1+\left(u_{1}-u_{A}\right)^{-}} \rightarrow 0$. 
By using the strong convergence: $u_{1} \rightarrow u_{A}$ in $L_{l o c}^{2}\left(\mathbb{R}^{2}\right)$ and the fact that $e^{u_{0}+u_{A}} \rightarrow 1$ as $|x| \rightarrow \infty$ (exponentially fast), we deduce that

$$
\left\|\left(u_{1}-u_{A}\right)^{+}\right\|_{L^{2}} \rightarrow 0 \text { and } \int_{\mathbb{R}^{2}}\left(\frac{\left(u_{1}-u_{A}\right)^{-}}{1+\left(u_{1}-u_{A}\right)^{-}}\right)^{2} \rightarrow 0 .
$$

So once more we can use (4.15) to conclude that $\left\|u_{1}-u_{A}\right\|_{L^{2}} \rightarrow 0$. Since the same strong convergence holds along any sequence, we conclude that, in the regime (4.8), $u_{1} \rightarrow u_{A}$ in $H^{1}\left(\mathbb{R}^{2}\right)$ as claimed.

\section{References}

[1] R. Auzzi, S. Bolognesi, J. Evslin, K. Konishi, Nonabelian monopoles and the vortices that confine them, Nucl. Phys. B 686 (2004), 119-134.

[2] R. Auzzi, S. Bolognesi, J. Evslin, K. Konishi, A. Yung, Nonabelian superconductors: vortices and confinement in $N=2 S Q C D$, Nucl. Phys. B 673 (2003), 187-216.

[3] M. Eto, T. Fujimori, S. B. Gunadson, K. Konishi, M. Nitta, K. Ohashi, W. Vinci, Constructing non-abelian vortices with arbitrary gauge groups, Phys. Lett. B 669 (2008), 98-101.

[4] M. Eto, T. Fujimori, T. Nagashima, M. Nitta, K. Okashi, N. Sakai, Multiple layer structure of non-abelian vortex, Phys. Lett. B 678 (2009), 254-258.

[5] M. Eto, Y. Isozumi, M. Nitta, K. Ohashi, N. Sakai, Moduli space of non-abelian vortices, Phys. Rev. Lett. 96 (2006), 161-601.

[6] M. Eto, Y. Isozumi, M. Nitta, K. Ohashi, N. Sakai, Solitons in Higgs phase - the moduli matrix approach, J. Phys. A 39 (2006), 315-392.

[7] S. B. Gunadson, Y. Jiang, K. Konishi, Non-abelian vortex dynamics: effective worldsheet action, J. High Energy Phys. 012 (2010). 1008

[8] A. Hanany, D. Tong, Vortex strings and four-dimensional gauge dynamics, J. High Energy Phys. 0404 (2004). 066

[9] A. Jaffe, C. H. Taubes, Vortices and Monopoles, Birkhäuser, Boston, 1980.

[10] K. Konishi, New results on non-abelian vortices - further insights into monopole, vortex and confinement, Int. J. Mod. Phys. A 25 (2010), 5025-5039.

[11] C. S. Lin, Y. Yang, Non-abelian multiple vortices in supersymmetric field theory, Commun. Math. Phys., to appear.

[12] C. S. Lin, Y. Yang, Sharp Existence and Uniqueness Theorems for non-abelian multiple vortex solutions, preprint 2010.

[13] A. Marshakov, A. Yung, Non-abelian confinement via abelian flux tubes in softly broken $N=2$ SUSY QCD , Nucl. Phys. B 647 (2002), 3-48.

[14] N. Sakai, D. Tong, Monopoles, vortices, domain walls and D-branes: the rules of interaction, J. High Energy Phys. 0503 (2005), 019.

[15] M. Shifman, M. Unsal, Confinement in Yang-Mills: elements of a big picture, Nucl. Phys. Proc. Suppl. 186 (2009), 235-242.

[16] M. Shifman, A. Yung, Non-abelian string junctions as confined monopoles, Phys. Rev. D 70 (2004), 045004.

[17] D. Tong, Monopoles in the Higgs phase, Phys. Rev. D 69 (2004), 065003. 
[18] G. Tarantello, Selfdual gauge Field vortices: an analytical approach, PNLDE 72, Birkhäuser, Boston, 2008.

[19] G. Tarantello, On some Elliptic problems in the study of selfdual Chern-Simons vortices, Geometrical Analysis and PDE, Chang et al. (eds), Lecture Notes in Mathematcis 1977 (2009), 117-175, Springer-Verlag, Berlin, Heidelberg .

Gabriella Tarantello

Universitá di Roma Tor Vergata

Via della Ricerca Scientifica snc

00133 Rome

Italy

e-mail: tarantel@mat.uniroma2.it

Received: March 3, 2011.

Open Access This article is distributed under the terms of the Creative Commons Attribution Noncommercial License which permits any noncommercial use, distribution, and reproduction in any medium, provided the original author(s) and source are credited. 